\title{
BIOLOGIA DE Conotrachelus humeropictus, FIEDLER, 1940 (COLEOPTERA: CURCULIONIDAE), PRAGA DO CACAUEIRO E DO CUPUAÇUZEIRO NA AMAZÔNIA BRASILEIRA'.
}

\author{
Antonio C. de B. MENDES ${ }^{2}$, Bonifácio P. MAGALHÃES ${ }^{3}$, Orlando S. OHASHI ${ }^{4}$
}

RESUMO - Foram determinados diversos parâmetros da biologia de Conotrachelus humeropictus Fiedler, importante praga do cacaueiro, Theobroma cacao L. e do cupuaçuzeiro, T. grandiflorum (Will. ex Spreng.) Schum na Amazónia brasileira. A pesquisa foi realizada em laboratório, com fotofase natural de 12 horas, sob condições controladas de temperatura $(27 \pm$ $\left.2^{\circ} \mathrm{C}\right)$ e umidade relativa $(80 \pm 10 \%)$. C. humeropictus alimentado com folhas novas e frutos novos de cacueiros, apresentou em média, um ciclo de ovo a emergência do adulto do solo de 79 a 151 dias e em média, 108,80 $\pm 9,44$ dias $\left(X \pm E_{x 95}\right)$ para machos e 156,87 $\pm 16,19$ dias para fêmeas. O período de pré-oviposição foi de $16,20 \pm 0,49$ dias. O periodo de oviposição foi de $80,50 \pm 5,58$ dias, colocando cada fêmea, de 55 a 153 ovos, em média $108,45 \pm 6,21$ ovos, sendo as posturas diárias e constituidas em média por $1,29 \pm 0,03$ ovos. No interior dos frutos de cacau, o inseto necessitou de 4,73 $\pm 0,06$ dias e $26,64 \pm 0,17$ dias, para 0 desenvolvimento embrionário e larval, respectivamente. Após esse periodo, um total de 20,25 $\pm 1,50$ dias se passaram no solo, para o desenvolvimento das fases de pré-pupa $(6,07 \pm 0,06$ dias), pupa (9,62 $\pm 0,10$ dias) e completa formação fisiológica do adulto (4,56 $\pm 0,11$ dias). Neste interim, $C$. humeropictus torna-se mais vulnerável ao ataque de seus inimigos naturais, principalmente fungos, dentre os quais Metarhizium anisopliae (Metsch.) Sorok. e Beauveria bassiana (Bals.) Vuill., únicos inimigos constatados para a espécie no decorrer da pesquisa.

Palavras-Chave: Insecta, broca do cacau, broca do cupuaçu, Theobroma cacao, Theobroma grandiflorum.

Biology of Conotrachelus humeropictus Fiedler, 1940 (Coleoptera: Curculionidae), Pest of Cocoa and Cupuassu Fruits in Brazilian Amazon.

ABSTRACT - The biology of Conotrachelus humeropictus Fiedler, 1940, a serious pest of Theobroma cacao L. and T. grandiflorum (Will. ex Spreng.) Schum, were investigated. The research was conducted in laboratories under controled temperature $\left(27 \pm 2^{\circ} \mathrm{C}\right)$ and relative humidity $(80 \pm 10 \%)$ conditions, C. humeropictus fed on young leaves and fruits of cocoa. Its cycle from egg to adult emergency from soil, was estabilished in average from 79 to 151 days, being $108.80 \pm 9.44\left(\mathrm{X} \pm \mathrm{SE}_{95}\right)$ days for males and $156.87 \pm 16.19$ days for females. The preoviposition period was $16.20 \pm 0.49$ days. The oviposition period was $80.50 \pm 5.58$ days, when each female lays 55 to 153 eggs in average $108.45 \pm 6.21$ eggs, and the daily oviposition was $1.29 \pm 0.03$ eggs. For the larval and embrionaire phases inside the cocoa fruits, the insect needed 25 to 39 days, $(31.37 \pm 10.96$ days $)$. After this period, $20.25 \pm 1.50$ days in the soil were necessary for pre-pupal $(6,07 \pm 0,06$ days) and pupal $(9,62 \pm 0,10$ days $)$ development and completphysiologic adult formation $(4,56 \pm 0,11$ days). By this time, $C$. humeropictus becomes more vulnerable to attack of natural enemies, specially fungi Metarhizium anisopliae (Metsch.) Sorok and Beauveria bassiana (Bals.) Vuill. only enemies constated in the researche.

Key Words: Insecta, cocoa borer, cupuassu borer, Theobroma cacao, Theobroma grandiflorum.

\footnotetext{
Parte da tese de doutoramento do $1^{g}$ autor. Pesquisa parcialmente financiada pelo CNPq CEPLAC/ Superintendência Regional da Amazônia Oriental, C..P. 1801, 66.635-110, Belém, PA EMBRAPA/ CENARGEN, Caixa Postal, 02372, 70.849-970, Brasília, DF

4 FCAP/ Departamento de Biologia Vegetal e Fitossanidade, C.P. 917, 66.095-480, Belém, PA
} 


\section{INTRODUÇÃO}

A broca Conotrachelus humeropictus Fiedler é considerada uma das principais pragas do cacaueiro, Theobroma cacao L. na Amazônia brasileira, não só pelos prejuízos diretos causados aos frutos, como, principalmente, por alterar indiretamente as propriedades intrínsecas e extrínsecas das amêndoas do cacau, culminando com sua desclassificação para comercialização (MENDES et al., 1988). Sua incidência em frutos de cacaueiros em Ouro Preto d'Oeste, Rondônia, foi estimada em até $52 \%$, nas épocas mais favoráveis ao inseto (TREVISAN, 1969) e prejuizos em frutos da maioria das populações silvestres foram relatados (ALMEIDA et al., 1995). Ataca também o cupuaçuzeiro, $T$. grandiflorum (Will. ex Spreng.) Schum., tornando sua polpa, muito apreciada e utilizada sob a forma de sucos e doces, imprestável para o consumo (TREVISAN \& MENDES, 1991; VENTRURIERI, 1993). Perdas de até $50 \%$ dos frutos na safra de 1990 de Rondônia, em consequência de seus danos, foram relatadas por LAKER \& TREVISAN (1992).

Dados sobre a biologia e comportamento de importantes pragas do gênero Conotrachelus foram apresentados por vários autores (SLINGERLAND \& GROSBY, 1924; SNAPP, 1930; DENIER, 1942; PARADIS, 1956; SMITH, 1957; ARMSTRONG, 1958; SMITH \& FLESSEL, 1968; JACKLIN \& YONCE, 1970; YONCE et al., 1972; LEVINE \& HALL, 1977; BLANC et al., 1984; AMIS \& SNOW, 1985; TEDDERS \& PAYNE, 1986), sendo que para C. humeropictus essas informações são inexistentes, exceto alguns aspectos do seu comportamento no campo elucidados por TREVISAN (1989), que concluiu existir preferência da espécie em colocar seus ovos na metade inferior do fruto, aumentando essa preferência a partir dos dois meses de idade desse fruto. Conforme o autor, frutos localizados até um metro do solo, no tronco do cacaueiro, são menos atacados que os localizados em alturas superiores.

$\mathrm{O}$ desconhecimento da biologia de C. humeropictus tem se constituido obstáculo para condução e maior precisão de pesquisas em diversos campos da entomologia, principalmente aquelas direcionadas ao controle. Assim sendo, procurou-se com o presente trabalho, elucidar diversos parâmetros da biologia da espécie.

\section{MATERIAL E MÉTODOS}

Nos laboratórios da Sede Regional da Comissão Executiva do Plano da Lavoura Cacaueira - CEPLAC, em Belém, Pará, a temperatura de $27 \pm 2^{\circ} \mathrm{C}$ e umidade relativa de $80 \pm 10 \%$, foram confinados separadamente, 20 casais recém-emergidos e sexados conforme MENDES (1989) em tubos de PVC (10 $\mathrm{cm}$ de diâmetro $\times 20 \mathrm{~cm}$ de altura), contendo, em seu interior, folhas novas e frutos de cacaueiros com aproximadamente três meses de idade, como substrato alimentar e de postura. Os tubos apresentavam como fundo, placas de Petri com areia esterilizada e papel de filtro umedecidos diariamente com água destilada estéril. As extremidades superiores dos tubos foram fechadas com peças de tecidos de ny- 
lon (filó), presas com ligas de borracha. Esses casais foram formados a partir de larvas do último ínstar, coletadas no campo. Desse modo, determinaram-se os periodos de pré-oviposição e oviposição, total de ovos, por fêmea, e longevidade dos adultos. O total de adultos emergidos no laboratório permitiu a determinação da razão sexual.

Constatadas as posturas nos substratos e/ou papel filtro, estes eram renovados e os ovos separados e classificados por postura/dia/casal e acondicionados em caixas plásticas, hermeticamente fechadas e forradas com papel de filtro umedecido com água destilada esterilizada, para determinação do período de incubação e viabilidade dos ovos.

As larvas recém-eclodidas foram inoculadas individualmente em frutos de cacau com aproximadamente três meses de idade e transferidas a cada cinco dias para novos frutos, determinando-se, nessa fase, a duração do período larval e a viabilidade larval. Ao atingirem o último estádio, as larvas, naturalmente, abandonavam os frutos para iniciar o processo de pupação, sendo imediatamente transferidas, individualmente, para caixas plásticas contendo em seu interior mistura de solo esterilizado e vermiculita na proporção de 2:1, umedecidos com água destilada, conforme recomendado por AMIS \& SNOW (1985) para $C$. nenuphar Herbst. Assim, determinaramse os períodos de pré-pupa, pupa e a viabilidade pupal.

Os parâmetros biológicos foram estudados mediante leituras diárias no mesmo horário, com o auxilio, quando necessário, de microscópio estereoscópico. Com os dados obtidos, determinou-se a média $(\mathrm{X})$ e seu erro padrão $\left(\mathrm{E}_{\mathrm{x} 95}\right)$.

\section{RESULTADOS E DISCUSSÃO}

Os resultados referentes aos periodos das diversas fases do ciclo evolutivo de $C$. humeropictus, nas condições em que se desenvolveu a pesquisa, acham-se condensados na Tabela 1.

\section{Fase de ovo}

Em laboratório, as posturas de $C$. humeropictus foram efetuadas sobre vários locais: superfície interna das gaiolas utilizadas pelos casais, sobre o papel de filtro das placas, sob o tecido que servia de tampa, bem como nas folhas e frutos novos. Nestes frutos, os ovos foram introduzidos até um máximo de $3 \mathrm{~mm}$, no pericarpo. Logo após a postura, os ovos apresentavam uma

Tabela 1. Duração média e viabilidade dos diferentes estágios de Conotrachelus humeropictus em laboratório, à temperatua de $27 \pm 2^{\circ} \mathrm{C}$, umidade relativa de $80 \pm 10 \%$ e fotofase de 12 horas.

\begin{tabular}{lcccc}
\hline \multirow{2}{*}{ Estágio } & Número de & \multicolumn{3}{c}{ Duração (dias) } \\
\cline { 3 - 5 } & observações & Média $\pm E_{\times 95}$ & $\begin{array}{c}\text { Intervalo de } \\
\text { variaçăo }\end{array}$ & $\begin{array}{c}\text { Viabilidade } \\
(\%)\end{array}$ \\
\hline Ovo & 325 & $4,73 \pm 0,06$ & $4-6$ & 75,60 \\
Larval & 245 & $26,64 \pm 0,17$ & $21-33$ & 88,19 \\
Pré-Pupal & 215 & $6,07 \pm 0,06$ & $4-7$ & 99,59 \\
Pupal & 213 & $9,62 \pm 0,10$ & $8-12$ & 97,12 \\
Pré-adulto $^{\prime}$ & 205 & $4,56 \pm 0,11$ & $3-6$ & 99,51 \\
\hline
\end{tabular}

Morfologicamente formado e imóvel no solo. 
coloração branco-leitosa, tornando-se amarelo-palha, na medida em que se desenrolava o desenvolvimento embrionário. Sua forma é elíptica, com $0,26 \pm 0,01 \mathrm{~mm}$ de comprimento e 0,16 $\pm 0,01 \mathrm{~mm}$ de largura. O período embrionário foi de 04 a 06 dias com média de 4,73 dias; a viabilidade foi de $75,60 \%$, em média. Em condições naturais, é provável uma maior eclosão de larvas, já que não há manipulação e perda de água dos ovos no interior dos frutos.

\section{Fase de larva}

A larva recém-eclodida apresenta cerca de $0,81 \pm 0,02 \mathrm{~mm}$ de comprimento e coloração brancoleitosa. Ao atingir o máximo desenvolvimento larval, em média 26,64 dias da eclosão medindo 17,21 $\pm 0,34 \mathrm{~mm}$ de comprimento, coloração amarelada com cabeça de cor castanhoavermelhada e mandíbulas bem desenvolvidas, abandonou o fruto através da abertura de um orificio, indicando estar prestes a iniciar a fase de pupação. No campo, com este procedimento, a larva procura o solo para dar início a essa fase.

Durante o seu desenvolvimento, no interior do fruto, a larva passou por três ecdises, apresentando, portanto, quatro estádios. Não foi possível a determinação precisa do período de cada estádio, pois o manuseio das larvas destinadas para esse fim alterou o ciclo biológico do inseto.

TREVISAN (1989) constatou que larvas oriundas de posturas efetuadas em frutos com quatro meses de idade completaram todo o seu ciclo larval no interior do fruto, os quais apresentaram, inclusive, maior incidência de danos mecânicos. Entretanto, larvas de posturas realizadas em frutos com 4,5 meses não conseguiram se desenvolver, por não haver tempo suficiente, já que a maturação e a colheita ocorreram quando os frutos atingiram os cinco meses de idade, 30 dias após a postura no primeiro grupo de frutos. Assim, o período determinado em laboratório, acha-se bastante próximo ao que provavelmente ocorre no campo. Esse período é mais longo do que o da espécie C. nenuphar, em média de 10 a 11 dias, quando criadas em ameixa e pêssego, respectivamente (ARMSTRONG, 1958), e em média de 11 (PARADIS, 1956), 13 (SMITH, 1957) e 18,8 (AMIS \& SNOW, 1985), dias, em maçãs.

A viabilidade larval foi de $88,19 \%$. A mortalidade de $11,81 \%$, provavelmente foi decorrente do manuseio das larvas para a troca de alimento, já que não ocorreram patologias durante a criação no laboratório.

\section{Fase de pré-pupa}

Ao completar o desenvolvimento larval, C. humeropictus cessa sua alimentação e, abandonando o fruto, prepara a "câmara pupal" no solo e, cessando também seus movimentos, entra na fase pré-pupa, cujo período foi, em média, de 6,07 dias. Nessa fase a viabilidade foi de $99,59 \%$.

\section{Fase de pupa}

A pupa, do tipo exarada, quando recém-formada é branco-leitosa, tornando-se castanho-claro, à medida que se aproxima a eclosão do adulto. $\mathrm{O}$ período pupal foi de 08 a 12 dias, com duração média de 9,62 dias e a viabilidade pupal de $97,12 \%$. 


\section{Fase de adulto}

$\mathrm{O}$ adulto teneral, de cor castanhoclaro e $9,87 \pm 0,134 \mathrm{~mm}$ de comprimento permaneceu, por um período de 03 a 06 dias (média 4,56 dias), praticamente imóvel na antiga "câmara pupal" quando, então, $99,51 \%$ dos indivíduos emergiram do solo. A esse periodo PARADIS (1956) e SMITH (1957) para C. nenuphar e TEDDERS \& PAYNE (1986) para $C$. schoofi, denominaram de estádio imaginal e de pré-emergência do adulto, respectivamente.

Os machos emergiram do solo horas antes que as fêmeas e, quando colocados nas gaiolas, passaram a se alimentar das folhas novas e pedaços de frutos. A cópula ocorreu no mesmo dia da emergência, após a distribuição das fêmeas nas gaiolas e durou de 75 a 105 minutos (média 89,25 minutos) (Fig.1). Observaram-se casais copulando mais de uma vez, indicando que os machos são capazes de fecundar mais de uma fềmea. Este comportamento também foi constatado para as espécies $C$. nenuphar (SMITH, 1957) e C. schoofi (TEDDERS \& PAYNE, 1986).

Conforme a Tabela 2 , o período de prè-oviposição foi em média de 16 dias, próximo aos 13 dias assinalado por SMITH (1957) para C. nenuphar. As fềmeas viveram em média 41,70 dias a mais do que os machos, inclusive após a última postura e, da longevidade observada, uma média de 80,50 dias foram utilizados para postura. Esse período de vida do adulto foi menor que o constatado por GARCIA et al. (1988) em estudos de dispersão, quando adultos marcados no laboratório e liberados no campo foram recuperados até 180 dias após a liberação. É provável que a qualidade do alimento possa ter influenciado para um menor período de vida do adulto, uma vez que, na natureza, o inseto possui mais opções, não só de

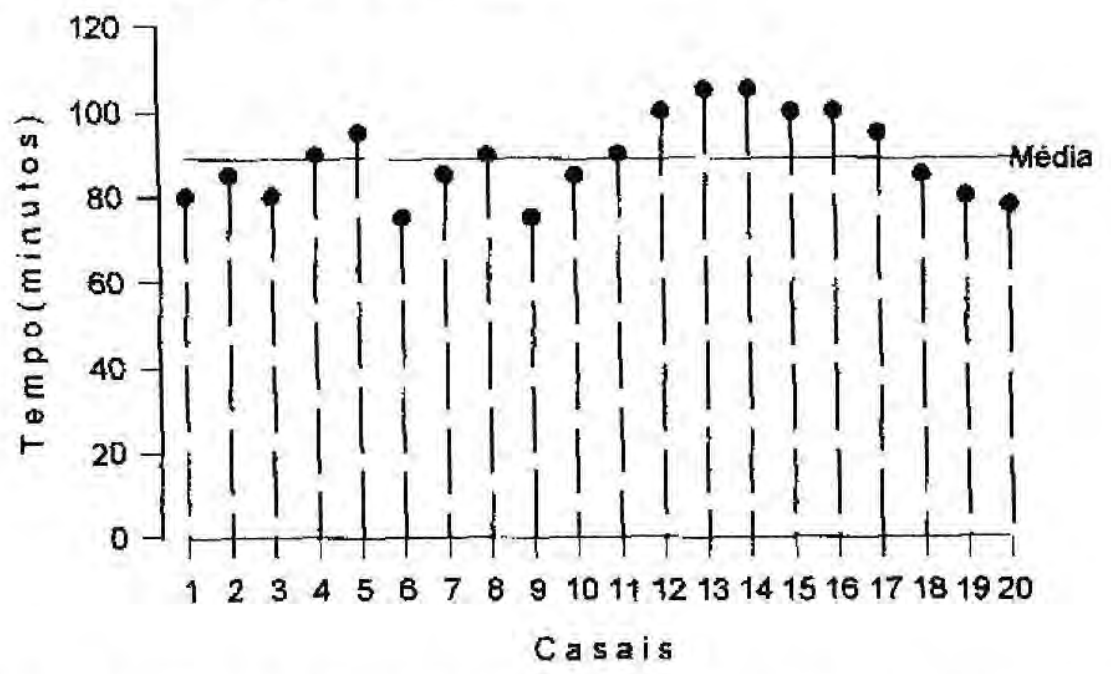

Figura 1. Tempo de cópula de Conotrachelus humeropictus em laboratório, à temperatura de $27 \pm 2^{\circ} \mathrm{C}$, umidade relativa de $80 \pm 10 \%$ e fotofase de 12 horas. 
Tabela 2. Períodos de pré-oviposição, oviposição número médio de ovos, pós-oviposição e longevidade no macho $(\sigma)$ e da fêmea ( $\$$ ) de Conotrachelus humeropictus em laboratório, à temperatura de $27 \pm 2^{\circ} \mathrm{C}$, umidade relativa de $80 \pm 10 \%$ e fotofase de 12 horas.

\begin{tabular}{|c|c|c|}
\hline \multirow{2}{*}{ Fase } & \multicolumn{2}{|c|}{ Duraçāo (dias) } \\
\hline & Média $\pm E_{x 95}$ & Intervalo de variação \\
\hline Pré-oviposição & $16,20 \pm 0,49$ & $11-20$ \\
\hline Oviposição & $80,50 \pm 5,58$ & $36-140$ \\
\hline Ovos/fêmea & $108,45 \pm 6,21$ & $55-153$ \\
\hline Ovos/fêmea/dia & $1,29 \pm 0,03$ & $01-2,5$ \\
\hline Pós-oviposição & $6,20 \pm 0,84$ & $01-13$ \\
\hline Longevidade $\sigma^{\prime}$ & $63,55 \pm 3,15$ & $39-87$ \\
\hline Longevidade & $105,25 \pm 5,88$ & $59-164$ \\
\hline
\end{tabular}

folhas e frutos novos de cacaueiros, como de outras espécies vegetais, já que foram constatados adultos se alimentando de frutos maduros de bananeira (Musa sp.) e de jaqueira (Artocarpus integrifolia L.).

As posturas de $C$. humeropictus foram realizadas de forma isolada, constatando-se posturas ocasionalmente constituídas por 02 a 03 e raramente de 04 ovos, colocando, cada fềmea, uma média de 108,45 ovos (Fig. 2).

$\mathrm{O}$ número médio de ovos depositados por fềmea/dia variou de $01 \mathrm{a}$ 2,5, com uma média geral de 1,29 ovos. O maior número de ovos foi colocado durante as primeiras posturas, entre o $18^{\circ}$ e 54을 dias da emergência (Fig. 3).

\section{Razão sexual}

A razão sexual observada foi de 0,49 o que demonstra uma relaçào de um macho para uma fêmea, a mesma encontrada para as espécies $C$. nenuphar (PARADIS, 1956; SMITH, 1957; ARMSTRONG, 1958) e C. schoofi ( TEDDERS \& PAYNE, 1986).

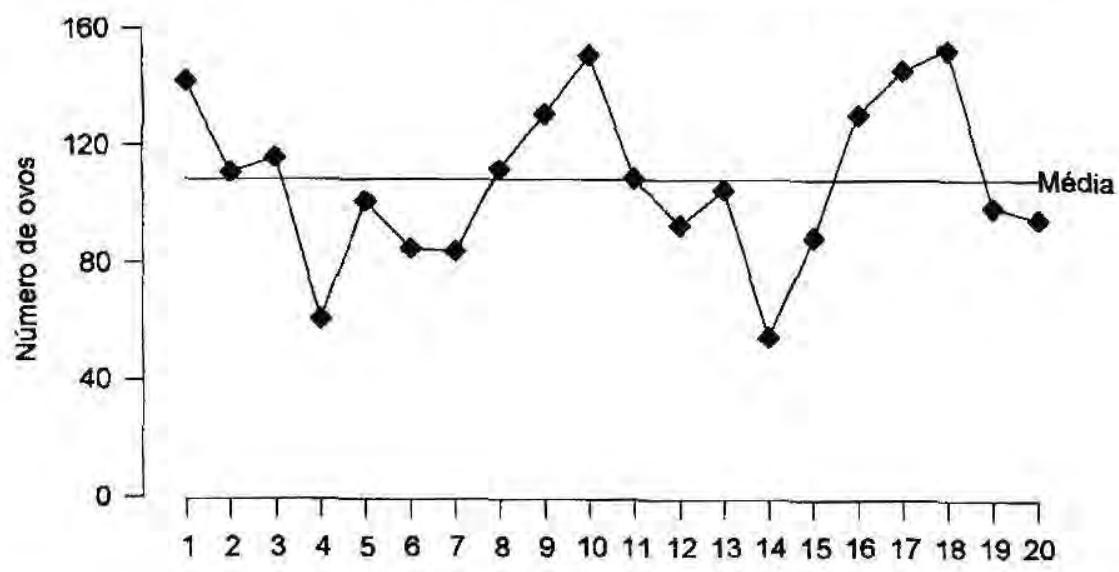

Fêmea

Figura 2. Número de ovos/fêmea de Conotrachelus humeropictus em laboratório, à temperatura de $27 \pm 2^{\circ} \mathrm{C}$, umidade relativa de $80 \pm 10^{\circ} \mathrm{C}$ e fotofase de 12 horas. 


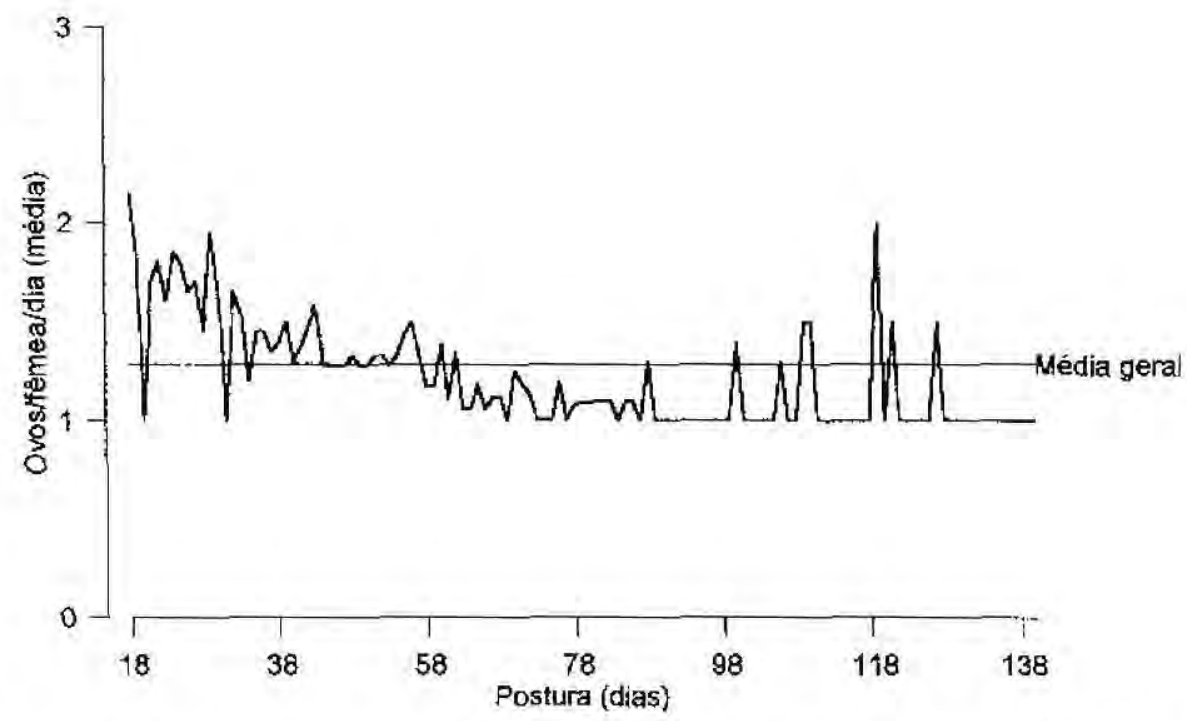

Figura 3. Número médio de ovos/fêmea/dia de Conotrachelus humeropictus em laboratório à temperatura de $27 \pm 2^{\circ} \mathrm{C}$, umidade relativa de $80 \pm 10^{\circ} \mathrm{C}$ e fotofase de 12 horas.

\section{Ciclo evolutivo de ovo a adulto}

O ciclo evolutivo médio de ovo a morte do adulto, foi de 108,80 dias para os machos e 156,87 dias, para as fềmeas. Desse ciclo, 31,37 dias foram utilizados para os periodos embrionário e larval que, na natureza, ocorrem no interior do fruto de Theobroma spp.. Para o desenvolvimento dos estágios de pré-pupa, pupa e amadurecimento fisiológico do adulto e sua saída do solo, foram necessários em média 20,25 dias, sendo essas, as fases mais vulneráveis do inseto, já que ovos e larvas ficam protegidos de seus inimigos naturais, no interior dos frutos (Tab. 3).

SMITH (1957), ARMSTRONG (1958) e JACKLIN \& YONCE (1970), assinalaram que a textura e umidade do solo constituem importantes fatores na mortalidade de $C$. nemuphar, constatando que a ausência prolongada de água no solo é responsável pela mortalidade de pré-pupas e pupas. Embora sendo espécie diferente, porém com hábitos bastante próximos ao besouro-da-ameixa, $C$. humeropictus também é influenciado pela umidade do solo, dai seu comportamento em desenvolver essas fases no solo durante o período chuvoso na região.

É interessante destacar que não se constatou parasitóides em 1687 larvas coletadas do interior de frutos de cacau e criadas em laboratório. Os únicos inimigos naturais observados foram os entomopatógenos Metarhizium anisopliae (Metsch.) Sorok. e Beauveria bassiana (Bals.) Vuill. (Fungi, Hyphomycetes), infectando pré-pupas no solo. Este fato, associado ao hábito de vida de $C$. humeropictus, justificam estudos para o seu controle através desses entomopatógenos.

\section{Número de gerações}

Nas condições em que foi desenvolvida a pesquisa, o ciclo de 
Tabela 3. Ciclo evolutivo de Conotrachelus humeropictus de ovo a adulto em laboratório, à temperatura de $27 \pm 2^{\circ} \mathrm{C}$ e umidade relativa de $80 \pm 10 \%$.

\begin{tabular}{ccc}
\hline \multirow{2}{*}{ Local de desenvolvimento } & \multicolumn{2}{c}{ Duração do ciclo (dias) } \\
\cline { 2 - 3 } & \multicolumn{1}{c}{ Média $\pm \mathrm{E}_{X 95}$} & Intervalo de variação \\
\hline Fruto & $31,37 \pm 10,96$ & $25-39$ \\
Solo & $20,25 \pm 1,50$ & $15-25$ \\
Exterior o & $63,55 \pm 3,15$ & $39-87$ \\
Exterior & $105,25 \pm 5,88$ & $59-16$ \\
$\sigma^{*}$ & $108,80 \pm 9,44$ & $79-151$ \\
우 & $156,87 \pm 16,17$ & $99-228$ \\
\hline
\end{tabular}

ovo a ovo apresentou um intervalo de variação de 110 a 248 e em média, 173 , 07 dias, o que equivale teoricamente à existência de uma a três e em média, duas gerações anuais da praga. Contudo, no habitat natural, a disponibilidade e qualidade do alimento, bem como fatores abióticos, certamente influenciam o número anual de gerações. AMIS \& SNOW (1985) assinalam a existência de duas formas de $C$. nenuphar, uma que necessita de diapausa para sua reprodução, denominada de raça setentrional, e outra que não apresenta diapausa, a raça meridional, bivoltina na natureza e multivoltina no laboratório.

\section{Bibliografia Citada}

ALMEIDA, C. M. V. C. de.; MACHADO, P. F. R. M; BARRIG A, J. P.; SILVA, F. C. O. da. 1995. Coleta de cacau (Theobroma cacao L.) da Amazônia Brasileira: uma abordagem histórica e analítica. Porto Velho: CEPLAC, Superintendência Regional da Amazônia Ocidental, 83p.

AMIS, A. A.; SNOW, J. W. 1985. Conotrachelus nenuphar. In: SING, P; MOORE, R. F. (Eds.). Handbook of Insect Rearing. Amsterdam: Elsevier, v.1, p.227-25.

ARMSTRONG, T. 1985. Life history and ecology of the plum curculio in the Niagara Penisula. Can. Entomol., v.90, p.8-17.
BLANC, J. P.; HILL, B. S.; PARADIS, R, D. 1984. Oviposition in scout-apples by plum curculio, Conotrachelus nenuphar (Herbst) (Coleoptera : Curculionidae) and its relationship to subsequent damage. Environ. Entomol., v.13, n.1, p.286-91.

DENIER, P. C. L. 1942. Apuntes sobre la biologia de Conotrachelus denieri Hust. plaga del agodonero. Rev. Soc. Entomol. Argentina, v.11, p.885-907.

GARCIA, J. de J, da S.; TREVISAN, O.; MENDES, A. C. de B. 1988. Estudo da migração de Conotrachelus humeropictus em lavouras de cacau em Ro. In: CEPLAC. Departamento Especial da Amazonia. Informe de Pesquisas 1987. Belén, p.28.

JACKLIN, S. W:; YONCE, C. E. 1970. Emergence, longevity, and fecundity of adult plum curculio reared from two sizes larvae at two temperature in soil with a range of misture. J. Econ. Entomol., v.63, p.637-74.

LAKER, H. A.; TREVISAN, O. 1992. The increasing importance of cupuassu (Theobroma grandiflorum) (Schum) in the Amazon Region of Brasil. Cocoa Growers' Bull., n.45, p. 45-52.

LEVINE, E; HALL, R, F. 1977. Effect of feeding and oviposition by the plum curculio on apple and plum fruit abscission. J. Econ. Entomol., v.70, p.603-607.

MENDES, A. C. de B.; RIBEIRO, N. C.; GARCIA, J. de J. da S.; TREVISAN, O. 1988. Danos de Conotrachelus humeropictus Fiedler, 1940 (Coleoptera : Curculionidae): Nova praga do cacaueiro (Theobroma cacao L.) na Amazônia 
brasileira. An. Soc. Entomol. Brasil., v.17, p.19-28.

MENDES, A. C. de B. 1989. Aspectos morfológicos de Conotrachelus humeropictus Fiedler, 1949 (Coleoptera : Curculionidae), In: CEPLAC, Coordenadoria de Pesquisas. Informe de Pesquisa 1988. Belém, p.18-19.

PARADIS, R, O. 1956, Observations sur le cycle évolutif du charançon de la prune, Conotrachelus nenuphar (Hbst.) sur la pomme dans le Québec. Ann. Entomol. Soc. Que., v.2, p.60-70.

SLINGERLAND, M. V; GROSBY, C. R, 1924, Quince insects, In: Slingerland, M. V. Manual of fruit ilnsects. New York: Millan, p.236-42.

SMITH, E. H. 1957. A method or rearing the plum curculio under laboratory conditions incluing some biological observations. J. Econ. Entomol. v.50, p.187-90.

SMITH, E. H.; FLESSEL, J. K. 1968. Hibernation of the plum curculio and its spring migration to host trees. J. Econ. Entomol., v.61, p. $193-203,1968$.

SNAPP, O. I. 1930. Life history and habits of the plum curculio the Georgia peach belt. $U$. S. Dept. Agri. Tech. Bull, 188, 76 p.
TEDDERS, W. L.; PAYNE, A. J. 1986. Biology, life history, and control of Conotrachelus schoofi (Coleoptera: Curculionidae) on pecans. J. Econ. Entomol, v.79, n.2, p, 490-96.

TREVISAN, O. 1989. Comportamento da broca dos frutos Conotrachelus humeropictus Fiedler, 1940 (Coleoptera : Curculionidae), em Rondónia. Piracicaba: Escola de Agricultura Luiz de Queiroz, 1989, 75p. Dissertaçào (Mestrado em Ciências Biológicas, Área de Entomologia). Universidade de São Paulo.

TREVISAN, O; MENDES, A. C. de B. 1991. Ocorrência de Conotrachelus humeropictus Fíedler, 1940 (Coleoptera : Curculionidae) em frutos de cupuaçu Theobroma grandifiorum (Willd, ex Spreng. \& Shum.) (Sterculiacea): CONGRESSO BRASILEIRO DE ENTOMOLOGIA, 13', Recife, Resumos. Recife: Sociedade Entomologica do Brasil, V.1, p.137.

VENTURIERI, G. A. 1993. Cupuaçu: a espécie, sua cultura, usos e pitscessamento. Belém: Clube do Cupu, $108 \mathrm{p}$.

YONCE, C. E.; PAYNE, J. A; PATE, R. R. 1972. Feeding and oviposition preferences of female plum curculio. $J$. Econ. Entomol., v.65, p.1206-07. 